\title{
A GENERALIZATION OF EULER NUMBERS TO FINITE COXETER GROUPS
}

\author{
MATTHIEU JOSUAT-VERGÈS
}

\begin{abstract}
It is known that Euler numbers, defined as the Taylor coefficients of the tangent and secant functions, count alternating permutations in the symmetric group. Springer defined a generalization of these numbers for each finite Coxeter group by considering the largest descent class, and computed the value in each case of the classification. We consider here another generalization of Euler numbers for finite Coxeter groups, building on Stanley's result about the number of orbits of maximal chains of set partitions. We present a method to compute these integers and obtain the value in each case of the classification.
\end{abstract}

\section{INTRODUCTION}

It is known since long ago [1] that the Euler numbers $T_{n}$, defined by

$$
\sec (z)+\tan (z)=\sum_{n \geq 0} T_{n} \frac{z^{n}}{n !}
$$

count alternating permutations in the symmetric group $\mathfrak{S}_{n}(\sigma$ is alternating if $\sigma(1)>$ $\sigma(2)<\sigma(3)>\ldots)$. Since then, there has been a lot of interest in these numbers and permutations, as exposed in the recent survey of Stanley [12].

It can be shown that alternating permutations form the largest descent class in the symmetric group. Building on this, Springer [11] gave a characterization of the largest descent class of a finite Coxeter group, and computed its cardinality in each case of the classification. The analog of alternating permutations for other groups were studied by Arnol'd [2], who called these objects snakes. See also [8, Section 3] for an alternative proof of Springer's result. Another relevant reference is Saito's article [10], where a more general problem is considered.

In this article, we are interested in another construction that relates the number $T_{n}$ with the symmetric group, and can also be generalized to finite Coxeter groups. Namely, there is an action of $\mathfrak{S}_{n}$ on the maximal chains in the lattice of set partitions of size $n$, and Stanley [13] showed that the number of orbits is $T_{n-1}$. It is now well established that set partitions can be realized as an intersection lattice generated by reflecting hyperplanes, so that the construction can be generalized and gives an integer $K(W)$ for each finite Coxeter group $W$, with $K\left(A_{n}\right)=T_{n}$. (Note that

2000 Mathematics Subject Classification. 05A18, 05E18, 11B68, $20 \mathrm{~F} 55$.

Key words and phrases. Euler numbers, Finite Coxeter groups, Set partitions.

Supported by ANR CARMA (ANR-12-BS01-0017). 
this differs from Springer's construction, where the the integer $T_{n}$ is related with the group $A_{n-1}$.) We present a general method to compute $K(W)$ and apply it to obtain the value in each case of the classification. There is some similarity with a problem studied by Reading [9], which consists in the enumeration of maximal chains in the lattice of noncrossing partition (in both cases, there is a product formula for the reducible case and a recursion on maximal parabolic subgroup in the irreducible case).

\section{ACKNOWLEDGEMENT}

I thank Vic Reiner for his helpful comments about this work. I also thank the anonymous referees for helpful suggestions and corrections.

\section{Definitions}

Let $V$ be a Euclidean space, and $W$ a finite subgroup of $G L(V)$ generated by orthogonal reflections. Let $n$ be the rank of $W$, i.e. $n=\operatorname{dim} V$. We call reflecting hyperplane an hyperplane $H \subset V$ which is the fixed point set of some reflection in $W$. The following definition is now well established, see for example [3, Chapter 4].

Definition 2.1. The set partition lattice $\mathcal{P}(W)$ is the set of linear subspaces of $V$ that are an intersection of reflecting hyperplanes. It is ordered by reverse inclusion, i.e. $\pi \leq \rho$ if $\rho \subset \pi$.

Remark 2.2. We are mostly interested in the case where $V$ is the standard geometric representation of a finite Coxeter group $W$. In this case, $\{0\} \in \mathcal{P}(W)$ and it is the maximal element. But in what follows, it will also be convenient to consider some reflection subgroup $U \subset W$. The definition is still valid and gives a subset $\mathcal{P}(U) \subset \mathcal{P}(W)$, and $\{0\} \notin \mathcal{P}(U)$ a priori.

In the case $A_{n}$ of the classification, $W$ is the symmetric group $\mathfrak{S}_{n+1}$ acting on $V=\left\{v \in \mathbb{R}^{n+1}: \sum v_{i}=0\right\}$ by permuting coordinates. The reflecting hyperplanes are $H_{i, j}=\left\{v \in V: v_{i}=v_{j}\right\}$ where $i<j$. We recover the traditional definition of a set partition, for example if $n=6$, then

$$
H_{1,7} \cap H_{2,4} \cap H_{4,5}=\left\{v \in V: v_{1}=v_{7}, v_{2}=v_{4}=v_{5}\right\} \in \mathcal{P}\left(A_{6}\right)
$$

corresponds to the set partition $17|245| 3 \mid 6$.

Let $t \in W$ be a reflection, and $H=\operatorname{Fix}(t)$ be its fixed point set. Then $w(H)=$ $\operatorname{Fix}\left(w t w^{-1}\right)$ for $w \in W$. So the natural action of $W$ on linear subspaces of $V$ gives an action of $W$ on the reflecting hyperplanes, and on $\mathcal{P}(W)$. Since inclusion and rank are preserved, this extends to an action on the maximal chains in $\mathcal{P}(W)$.

Definition 2.3. Let $\mathcal{M}(W)$ denote the set of maximal chains in $\mathcal{P}(W)$, i.e. sequences $C=\left(C_{0}, \ldots, C_{n}\right) \in \mathcal{P}(W)^{n+1}$ where $C_{0}<\cdots<C_{n}$ (this implies that $C_{i}$ has rank $i$ ). We define an integer $K(W)$ as the number of orbits for the $W$-action on $\mathcal{M}(W)$, i.e. $K(W)=\#(\mathcal{M}(W) / W)$. 
An element of $\mathcal{M}(W)$ can be seen as a complete flag of $V$. Thus we can rephrase the definition: $K(W)$ is the number of $W$-orbits of complete flags in $V$ where each element of the flag is a fixed point subspace of some $w \in W$.

Let us introduce further notations (see $[5,6]$ ). We recall that the complement in $V$ of the reflecting hyperplanes is divided into connected regions called chambers, and $W$ acts simply transitively on the chambers. Let $H_{1}, \ldots, H_{n}$ be the reflecting hyperplanes that enclose one particular chamber $R_{0}$, the fundamental chamber. Then the corresponding orthogonal reflections $s_{1}, \ldots, s_{n}$ form a set $S$ of simple generators for $W$. According to this choice, there is a longest element $w_{0}$ (the unique group element that maximizes the length function). For any $i$, let $W_{(i)} \subset W$ be the (standard maximal parabolic) subgroup generated by the $s_{j}$ with $j \neq i$. If $s \in S$, we also denote $W_{(s)}=W_{(i)}$ if $s=s_{i}$. An alternative description is that, if we define a line

$$
L_{i}=\bigcap_{\substack{1 \leq j \leq n \\ j \neq i}} H_{j},
$$

then $w \in W_{(i)}$ if and only if $w(v)=v$ for any $v \in L_{i}$. The lines $L_{i}$ are exactly those in $\mathcal{P}(W)$ that are incident to the fondamental chamber $R_{0}$.

For each line $L \in \mathcal{P}(W)$, we define two subgroups of $W$, respectively the stabilizer and the pointwise stabilizer:

$$
\begin{aligned}
\operatorname{Stab}(L) & =\{w \in W: w(L)=L\}, \\
\operatorname{Stab}^{*}(L) & =\{w \in W: \forall x \in L, w(x)=x\} .
\end{aligned}
$$

Note that $\operatorname{Stab}^{*}(L)$ is a subgroup of $\operatorname{Stab}(L)$ with index either 1 or 2 . The group $\operatorname{Stab}^{*}(L)$ is generated by the reflections it contains and is itself a real reflection group, its reflecting hyperplanes being those of $W$ containing $L$. So we can identify $\mathcal{P}\left(\operatorname{Stab}^{*}(L)\right)$ with the interval $[V, L] \subset \mathcal{P}(W)$.

\section{The GEneral Method}

We describe how the integer $K(W)$ can be computed inductively. To begin, in the reducible case we have:

Proposition 3.1. Let $W_{1}$ and $W_{2}$ be two Coxeter groups of respective ranks $m$ and $n$, then

$$
K\left(W_{1} \times W_{2}\right)=\left(\begin{array}{c}
m+n \\
m
\end{array}\right) K\left(W_{1}\right) K\left(W_{2}\right) .
$$

Proof. First, note that there is natural identification $\mathcal{P}\left(W_{1}\right) \times \mathcal{P}\left(W_{2}\right)=\mathcal{P}\left(W_{1} \times W_{2}\right)$. The idea is to shuffle an element of $\mathcal{M}\left(W_{1}\right)$ with one of $\mathcal{M}\left(W_{2}\right)$ and the details are as follows. Let $\left(x_{0}, \ldots, x_{m}\right) \in \mathcal{M}\left(W_{1}\right)$ and $\left(y_{0}, \ldots, y_{n}\right) \in \mathcal{M}\left(W_{2}\right)$. By elementary properties of the product order, we can form an element $C \in \mathcal{M}\left(W_{1} \times W_{2}\right)$ by considering a sequence

$$
C=\left(\left(x_{i_{0}}, y_{j_{0}}\right), \ldots,\left(x_{i_{m+n}}, y_{j_{m+n}}\right)\right)
$$


where the indices are such that $i_{0}=j_{0}=0, i_{m+n}=m, j_{m+n}=n$, and for $0 \leq k<$ $m+n$ :

- either $i_{k+1}=i_{k}$ and $j_{k+1}=j_{k}+1$,

- or $i_{k+1}=i_{k}+1$ and $j_{k+1}=j_{k}$.

If $I$ denotes the set of possible choices for the indices $i_{k}$ and $j_{k}$, this defines a bijection

$$
I \times \mathcal{M}\left(W_{1}\right) \times \mathcal{M}\left(W_{2}\right) \rightarrow \mathcal{M}\left(W_{1} \times W_{2}\right) .
$$

Since the bijection commutes with the action of $W_{1} \times W_{2}$ and $\# I=\left(\begin{array}{c}m+n \\ m\end{array}\right)$, the result is proved.

We suppose now that $W$ is irreducible. A natural approach to find $K(W)$ is to distinguish the maximal chains according to the coatom they contain (in terms of complete flags, we distinguish them according to the line they contain). Doing the same thing at the level of orbits will lead to Proposition 3.2 below.

Recall that we can identify $\mathcal{P}\left(\operatorname{Stab}^{*}(L)\right)$ with $[V, L] \subset \mathcal{P}(W)$. There is also a natural way to see $\mathcal{M}\left(\operatorname{Stab}^{*}(L)\right)$ as a subset of $\mathcal{M}(W)$, namely $\left(C_{0}, \ldots, C_{n-1}\right) \in$ $\mathcal{M}\left(\operatorname{Stab}^{*}(L)\right)$ is identified with $\left(C_{0}, \ldots, C_{n-1},\{0\}\right)$. Clearly, $[V, L]$ is stable by the action of $\operatorname{Stab}(L)$ and this extends to an action of $\operatorname{Stab}(L)$ on $\mathcal{M}\left(\operatorname{Stab}^{*}(L)\right)$. With this at hand, we have:

Proposition 3.2. Let $\mathcal{L} \subset \mathcal{P}(W)$ be a set of orbit representatives for the action of $W$ on lines in $\mathcal{P}(W)$, then:

$$
K(W)=\sum_{L \in \mathcal{L}} \#\left(\mathcal{M}\left(\operatorname{Stab}^{*}(L)\right) / \operatorname{Stab}(L)\right) .
$$

Proof. If $C=\left(C_{0}, \ldots, C_{n}\right) \in \mathcal{M}(W)$, there is a unique $L \in \mathcal{L}$ such that the coatom $C_{n-1}$ and $L$ are in the same $W$-orbit. Moreover, $L$ only depends on the $W$-orbit of $C$, so this defines a map $f: \mathcal{M}(W) / W \rightarrow \mathcal{L}$.

With the discussion above in mind, we identify $\mathcal{M}\left(\operatorname{Stab}^{*}(L)\right)$ with the set of chains $C=\left(C_{0}, \ldots, C_{n}\right) \in \mathcal{M}(W)$ satisfying $C_{n-1}=L$. Each element of $f^{-1}(L)$ is a $W$-orbit that can be represented by an element of $\mathcal{M}\left(\operatorname{Stab}^{*}(L)\right)$, and two elements of $\mathcal{M}\left(\operatorname{Stab}^{*}(L)\right)$ are in the same $W$-orbit if and only if they are in the same $\operatorname{Stab}(L)$ orbit. This permits to define a bijection between $f^{-1}(L)$ and $\mathcal{M}\left(\operatorname{Stab}^{*}(L)\right) / \operatorname{Stab}(L)$. Now, we can write:

$$
K(W)=\#(\mathcal{M}(W) / W)=\sum_{L \in \mathcal{L}} \#\left(f^{-1}(L)\right)=\sum_{L \in \mathcal{L}} \#\left(\mathcal{M}\left(\operatorname{Stab}^{*}(L)\right) / \operatorname{Stab}(L)\right),
$$

as announced.

Now, let us describe how to find the set $\mathcal{L}$ of orbit representatives for the action of $W$ on lines in $\mathcal{P}(W)$. We can use the lines $L_{i}$ defined in Equation (2) from the previous section.

Proposition 3.3. Each line $L \in \mathcal{P}(W)$ can be written $w\left(L_{i}\right)$ for some $w \in W$ and $1 \leq i \leq n$. If $w \in W$ and $i \neq j$, then $w\left(L_{i}\right)=L_{j}$ implies $w_{0}\left(L_{i}\right)=L_{j}$. 
Similar considerations appeared in the work of Armstrong, Reiner and Rhoades [4], in the context of $W$-parking functions. Still, it is reasonable to include a short proof here.

Proof. Let us split the line $L$ in two half-lines $L^{+}$and $L^{-}$, and let $R$ be a chamber incident to $L^{+}$. We also split $L_{i}$ in two half-lines $L_{i}^{+}$and $L_{i}^{-}$, where $L_{i}^{+}$is the one incident to $R_{0}$. The group $W$ acts simply transitively on the chambers, so there is $w \in W$ such that $w\left(R_{0}\right)=R$. Then $w^{-1}\left(L^{+}\right)$is incident to $R_{0}$, so there is $i$ such that $w^{-1}\left(L^{+}\right)=L_{i}^{+}$, and consequently $L^{+}=w\left(L_{i}^{+}\right)$and $L=w\left(L_{i}\right)$.

Now, suppose we have $i \neq j$ and $w\left(L_{i}\right)=L_{j}$. We have either $w\left(L_{i}^{+}\right)=L_{j}^{+}$or $w\left(L_{i}^{+}\right)=L_{j}^{-}$(where $L_{j}^{+}$and $L_{j}^{-}$are defined in the same way as with $L_{i}$ ). In the first case, $R_{0}$ and $w\left(R_{0}\right)$ are both incident to $L_{j}^{+}$. This implies $w\left(L_{j}^{+}\right)=L_{j}^{+}$(note that $W_{(j)}$ acts simply transitively on the set of chambers incident to $L_{j}^{+}$), but this is a contradiction with $i \neq j$ and $w\left(L_{i}\right)=L_{j}$. So we have $w\left(L_{i}^{+}\right)=L_{j}^{-}$. Since $L_{j}^{-}$ is incident to both $-R_{0}$ and $w\left(R_{0}\right)$, there is $u \in W_{(j)}$ such that $u w\left(R_{0}\right)=-R_{0}$, i.e. $u w=w_{0}$. Then, we have $w_{0}\left(L_{i}^{+}\right)=u w\left(L_{i}^{+}\right)=u\left(L_{j}^{-}\right)=L_{j}^{-}$. So $w_{0}\left(L_{i}\right)=L_{j}$.

From the definition of $L_{i}$ in Equation (2), $w_{0}\left(L_{i}\right)=L_{j}$ is equivalent to $w_{0}\left(H_{i}\right)=$ $H_{j}$, which is also equivalent to $w_{0} s_{i} w_{0}=s_{j}$. Elementary properties of the longest element show that the map defined on the simple generators by $s \mapsto w_{0} s w_{0}$ is an involutive automorphism of the Coxeter graph. One can also show that this automorphism is the identity if and only if the exponents of the group are all odd, see [5, Exercise 4.10]. So the set $\mathcal{L}$ can be obtained by taking $\left\{L_{1}, \ldots, L_{n}\right\}$, quotiented by the action of $w_{0}$ which can be described in a precise way.

We have Stab* $\left(L_{i}\right)=W_{(i)}$, the standard maximal parabolic subgroup. To identify the group $\operatorname{Stab}\left(L_{i}\right)$, we have the following:

Proposition 3.4. Either $\operatorname{Stab}\left(L_{i}\right)=W_{(i)}$, or $\operatorname{Stab}\left(L_{i}\right)=<W_{(i)}, w_{0}>$.

Proof. Suppose there is $w \in \operatorname{Stab}\left(L_{i}\right)$ with $w \notin W_{(i)}$, which means that $w\left(L_{i}^{+}\right)=L_{i}^{-}$. So $w\left(R_{0}\right)$ is incident to $L_{i}^{-}$. Since $W_{(i)}$ acts transitively on the chambers incident to $L_{i}^{-}$, there is $u \in W_{(i)}$ with $u w\left(R_{0}\right)=-R_{0}$, i.e. $u w=w_{0}$. It follows $w_{0} \in \operatorname{Stab}\left(L_{i}\right)$ with $w_{0} \notin W_{(i)}$.

Since $W_{(i)}$ has rank $n-1$, by induction we can assume we already know the integer $K\left(W_{(i)}\right)$, which is useful in some situations.

Proposition 3.5. With $W, w_{0}$, and $L_{i} \in \mathcal{L}$ as above, we have:

- If $w_{0} s_{i} w_{0} \neq s_{i}$, then

$$
\#\left(\mathcal{M}\left(W_{(i)}\right) / \operatorname{Stab}\left(L_{i}\right)\right)=K\left(W_{(i)}\right) .
$$

- If $w_{0} s_{i} w_{0}=s_{i}$, and there is $u \in W_{(i)}$ such that $w_{0} s_{j} w_{0}=u s_{j} u$ for any $j \neq i$, then

$$
\#\left(\mathcal{M}\left(W_{(i)}\right) / \operatorname{Stab}\left(L_{i}\right)\right)=K\left(W_{(i)}\right) .
$$


- If $w_{0} s_{i} w_{0}=s_{i}$, and the map $s \mapsto w_{0} s w_{0}$ permutes nontrivially the connected components of the Coxeter graph of $W_{(i)}$, then:

$$
\#\left(\mathcal{M}\left(W_{(i)}\right) / \operatorname{Stab}\left(L_{i}\right)\right)=\frac{1}{2} K\left(W_{(i)}\right) .
$$

Proof. If $w_{0} s_{i} w_{0} \neq s_{i}$, then $w_{0} \notin \operatorname{Stab}\left(L_{i}\right)$, hence $\operatorname{Stab}\left(L_{i}\right)=W_{(i)}$ using Proposition 3.4. This proves the first point.

Suppose $w_{0} s_{i} w_{0}=s_{i}$ and there exists $u$ as above. It means that the action of $u$ on $\mathcal{M}\left(W_{(i)}\right)$ is the same as the action of $w_{0}$. In either of the two cases given in Proposition 3.4, we find that the $\operatorname{Stab}\left(L_{i}\right)$-orbits are exactly the $W_{(i)}$-orbits. This proves the second point.

As for the third point, we suppose there are only two connected components in the Coxeter graph of $W_{(i)}$, the general case being similar. Let us write $W_{(i)}=$ $W_{1} \times W_{2}$. We have seen in the proof of Proposition 3.1 that the elements of $\mathcal{M}\left(W_{(i)}\right)$ are obtained by "shuffling" two elements of $\mathcal{M}\left(W_{1}\right)$ and $\mathcal{M}\left(W_{2}\right)$. So if $C=\left(C_{0}, \ldots, C_{n-1}\right) \in \mathcal{M}\left(W_{(i)}\right)$, the element $C_{1}$ is a pair $\left(C_{1}^{\prime}, C_{1}^{\prime \prime}\right) \in \mathcal{P}\left(W_{1}\right) \times \mathcal{P}\left(W_{2}\right)$ where the respective ranks of $C_{1}^{\prime}$ and $C_{1}^{\prime \prime}$ are either 0 and 1 , or 1 and 0 . These two conditions are preserved by the action of $W_{(i)}$, and are reversed by the action of $w_{0}$. So the action of $w_{0}$ on $\mathcal{M}\left(W_{(i)}\right) / W_{(i)}$ has no fixed point and each orbit has cardinality 2 . We can write:

$$
\#\left(\mathcal{M}\left(W_{(i)}\right) / \operatorname{Stab}\left(L_{i}\right)\right)=\#\left(\left(\mathcal{M}\left(W_{(i)}\right) / W_{(i)}\right) / w_{0}\right)
$$

and this proves the result.

Let us summarize the situation. If $w_{0}$ is central in $W$, we can always apply the second case of Proposition 3.5, so that Proposition 3.2 gives

$$
K(W)=\sum_{s \in S} K\left(W_{(s)}\right)
$$

where each $W_{(s)}$ is a standard maximal parabolic subgroup of $W$. Furthermore, some of the terms are simplified using the product formula in Proposition 3.1. In particular, this equation can be directly obtained from the Coxeter graph.

When $w_{0}$ is not central, the map $s \mapsto w_{0} s w_{0}$ is an involution on the set $S$ of simple generators and we need to distinguish the two-element orbits and the fixed points. Indeed, we have:

$$
K(W)=\sum_{\substack{\left\{s_{i}, s_{j}\right\} \subset S, s_{i} \neq s_{j} \\ w_{0} s_{i} w_{0}=s_{j}}} K\left(W_{(i)}\right)+\sum_{\substack{s_{i} \in S \\ w_{0} s_{i} w_{0}=s_{i}}} \#\left(\mathcal{M}\left(W_{(i)}\right) / \operatorname{Stab}\left(L_{i}\right)\right) .
$$

Some terms in the first sum (respectively, the second sum) can be further simplified using Proposition 3.1 (respectively, Proposition 3.5).

Note that Proposition 3.5 does not exhaust all the possibilities, so we do not have a general solution to find all the terms $\#\left(\mathcal{M}\left(W_{(i)}\right) / \operatorname{Stab}\left(L_{i}\right)\right)$ in the second sum 
of Equation (4). As we will see in the next section, the only case that cannot be treated directly will appear when $W=D_{n}$ with $n$ odd.

\section{The CASe By CASE Resolution}

We follow the traditional notation for the classification of finite irreducible Coxeter groups, see [5]. We will denote $a_{n}=K\left(A_{n}\right), b_{n}=K\left(B_{n}\right), d_{n}=K\left(D_{n}\right)$. It will be convenient to take the conventions that $A_{0}=B_{0}=D_{0}$ (the trivial group with rank 0), $A_{1}=B_{1}, D_{2}=A_{1} \times A_{1}$ and $D_{3}=A_{3}$.

Proposition 4.1 (See [5], Exercise 4.10). In the groups $I_{2}(m)$ for $m$ even, $B_{n}, D_{n}$ for $n$ even, $G_{2}, H_{3}, H_{4}, E_{7}$, and $E_{8}$, the longest element is central. In the other groups, i.e. $I_{2}(m)$ for $m$ odd, $A_{n}, D_{n}$ for $n$ odd, and $E_{6}$, the map $s \mapsto w_{0} s w_{0}$ is the unique nontrivial automorphism of the Coxeter graph.

4.1. Case of $A_{n}$. We already know that $a_{n}=T_{n}$, but let us check how to prove it with our method. Here, $w_{0}$ is not central and $s \mapsto w_{0} s w_{0}$ reverses the $n$ vertices of the Coxeter graph. There is a fixed point only if $n$ is odd, and it can be treated using the third case of Proposition 3.5. So Equation (4) gives, when $n \geq 2$ :

$$
a_{n}=\sum_{i=0}^{\lfloor n / 2\rfloor-1}\left(\begin{array}{c}
n-1 \\
i
\end{array}\right) a_{i} a_{n-1-i}+[n \bmod 2] \times \frac{1}{2}\left(\begin{array}{c}
n-1 \\
(n-1) / 2
\end{array}\right) a_{(n-1) / 2}^{2} .
$$

(Here, $[n \bmod 2]$ is considered as the natural number 0 or 1.) This can be rewritten as:

$$
a_{n}=\frac{1}{2} \sum_{i=0}^{n-1}\left(\begin{array}{c}
n-1 \\
i
\end{array}\right) a_{i} a_{n-1-i}
$$

Let us define

$$
A(z)=\sum_{n \geq 0} a_{n} \frac{z^{n}}{n !}
$$

Multiplying Equation (5) by $\frac{z^{n-1}}{(n-1) !}$ and summing over $n \geq 2$ gives

$$
A^{\prime}(z)-1=\frac{1}{2}\left(A(z)^{2}-1\right)
$$

So $A(z)$ is the solution of the differential equation $A^{\prime}(z)=\frac{1}{2}\left(A(z)^{2}+1\right)$ with the initial value $A(0)=1$. It can be checked that $A(z)=\tan (z)+\sec (z)$ is the solution, so that $a_{n}=T_{n}$.

4.2. Case of $B_{n}$. In this group, the longest element is central. Equation (3) together with the product formula gives:

$$
b_{n}=\sum_{i=0}^{n-1}\left(\begin{array}{c}
n-1 \\
i
\end{array}\right) b_{i} a_{n-i-1} .
$$


Now, let

$$
B(z)=\sum_{n \geq 0} b_{n} \frac{z^{n}}{n !}
$$

Multiplying Equation (6) by $\frac{z^{n-1}}{(n-1) !}$ and summing over $n \geq 1$ gives

$$
B^{\prime}(z)=B(z) A(z) \text {. }
$$

So $B(z)$ is the solution of the differential equation $B^{\prime}(z)=B(z) A(z)$ with initial value $B(0)=1$. We can check that

$$
B(z)=\frac{1}{1-\sin (z)}
$$

is a solution. This function also satisfies $B(z)=A^{\prime}(z)$, so that

$$
b_{n}=T_{n+1} .
$$

A bijective proof of this will be given in [7].

4.3. Case of $D_{n}$. When $n$ is even, the longest element of $D_{n}$ is central and Equation (3) gives:

$$
d_{n}=2 a_{n-1}+\sum_{2 \leq i \leq n-1}\left(\begin{array}{c}
n-1 \\
i
\end{array}\right) d_{i} a_{n-1-i} .
$$

In the case when $\mathrm{n}$ is odd, one cannot quite write the equation as immediately. The map $s \mapsto w_{0} s w_{0}$ exchanges two vertices of the Coxeter graph, and this gives one term $a_{n-1}$ coming from the first sum in Equation (4). As for the second sum, we are in the case where $s_{i}=w_{0} s_{i} w_{0}$, and $W_{(i)}=D_{i} \times A_{n-1-i}$. If $i$ is odd, we can apply the second case of Proposition 3.5 where $u$ is chosen to be the longest element of the factor $D_{i}$. More care is needed when $i$ is even, i.e. when we cannot directly apply Proposition 3.5. So we consider the set $\mathcal{M}\left(D_{i} \times A_{n-1-i}\right)$, quotiented by $D_{i} \times A_{n-1-i}$, and further quotiented by the graph automorphism of the factor $D_{i}$ (the graph automorphism induces an action on $\mathcal{P}\left(D_{i}\right)$ ). An argument similar to the one in Proposition 3.1 shows that the number of orbits can be factorized. Eventually, we obtain:

$$
d_{n}=a_{n-1}+\sum_{\substack{2 \leq i \leq n-1 \\
i \text { odd }}}\left(\begin{array}{c}
n-1 \\
i
\end{array}\right) d_{i} a_{n-1-i}+\sum_{\substack{2 \leq i \leq n-1 \\
i \text { even }}}\left(\begin{array}{c}
n-1 \\
i
\end{array}\right) \bar{d}_{i} a_{n-1-i}
$$

where $\bar{d}_{i}$ is defined as follows: it is the number of orbits for the action on $\mathcal{M}\left(D_{i}\right)$ generated by $D_{i}$ together with the graph automorphism (except that if $i=4$, the graph automorphism is not unique but we only consider the one that exchanges two vertices). Note that for odd $i$, we can define $\bar{d}_{i}$ similarly but it is clear that $\bar{d}_{i}=d_{i}$. We need to compute $\bar{d}_{n}$ before solving the recursion for $d_{n}$. 
Proposition 4.2. We have $\bar{d}_{0}=1$ and for any $n \geq 1$,

$$
\bar{d}_{n}=a_{n-1}+\sum_{i=2}^{n-1}\left(\begin{array}{c}
n-1 \\
i
\end{array}\right) \bar{d}_{i} a_{n-1-i} .
$$

Proof. Although we cannot directly apply Proposition 3.2 and Proposition 3.1, the argument is completely similar, so we omit details. Let $\Gamma$ denote the graph automorphism of $D_{n}$.

Suppose $L_{1}$ and $L_{2}$ are the two coatoms that are exchanged by $\Gamma$. Counting orbits of maximal chains having $L_{1}$ or $L_{2}$ as coatom, we obtain the first term $a_{n-1}$.

If $i \neq 1,2$, the number of orbits of maximal chains having $L_{i}$ as coatom is the number of orbits in $\mathcal{M}\left(W_{(i)}\right) /<\operatorname{Stab}\left(L_{i}\right), \Gamma>$. This is also the number of orbits in $\mathcal{M}\left(W_{(i)}\right) /<W_{(i)}, \Gamma>$, since either $\operatorname{Stab}\left(L_{i}\right)=W_{(i)}$ or $\operatorname{Stab}\left(L_{i}\right)=<W_{(i)}, w_{0}>$ where $w_{0}$ has the same action as $\Gamma$. We have a decomposition $W_{(i)}=D_{i} \times A_{n-1-i}$ and the graph automorphism only acts on the factor $D_{i}$. So the argument of Proposition 3.1 shows that this number is $\bar{d}_{i} a_{n-1-i}$.

Proposition 4.3. If $n \geq 2$, we have $\bar{d}_{n}=2 a_{n+1}-(n+1) a_{n}$.

Proof. The recursion in the previous proposition shows that the generating function $\bar{D}(z)=\sum_{n \geq 0} \bar{d}_{n} \frac{z^{n}}{n !}$ satisfies the differential equation

$$
\bar{D}^{\prime}(z)=(\bar{D}(z)-z) A(z),
$$

with the initial condition $\bar{D}(0)=1$. This is solved by

$$
\bar{D}(z)=\frac{2-\cos (z)-z \sin (z)}{1-\sin (z)} .
$$

From this expression, we can get $\bar{D}(z)=(2-z) A^{\prime}(z)+z-A(z)$, and it follows that $\bar{d}_{n}=2 a_{n+1}-(n+1) a_{n}$ if $n \geq 2$.

Proposition 4.4. Let $n \geq 2$. We have $d_{n}-\bar{d}_{n}=a_{n}$ if $n$ is even, and $d_{n}=\bar{d}_{n}$ otherwise.

Proof. If $n \geq 2$, from (7), (8), and (9), we have:

$$
d_{n}-\bar{d}_{n}=\chi[n \text { even }] \times a_{n-1}+\sum_{\substack{2 \leq i \leq n-1 \\
n-i \text { even }}}\left(\begin{array}{c}
n-1 \\
i
\end{array}\right)\left(d_{i}-\bar{d}_{i}\right) a_{n-1-i} .
$$

Here and in the sequel, $\chi$ means 1 or 0 depending on whether the condition within brackets is true or false. So the generating function

$$
U(z)=1+\sum_{n \geq 2}\left(d_{n}-\bar{d}_{n}\right) \frac{z^{n}}{n !}
$$

satisfies $U^{\prime}(z)=U(z) \tan (z)$ and $U(0)=1$. This is solved by $U(z)=\sec (z)$ and the result follows. 
From the previous two propositions, we get that for $n \geq 2$,

$$
d_{n}= \begin{cases}2 T_{n+1}-n T_{n} & \text { if } n \text { is even, } \\ 2 T_{n+1}-(n+1) T_{n} & \text { if } n \text { is odd } .\end{cases}
$$

From (10), we can separate the odd and even parts of $\bar{D}(z)$ (multiply the numerator and denominator by $1+\sin (z)$ and separate terms in the numerator). After some calculation, this leads to:

$$
\sum_{n \geq 1} d_{2 n} \frac{z^{2 n}}{(2 n) !}=\frac{\sin (z)(2 \sin (z)-z)}{\cos (z)^{2}}
$$

and

$$
\sum_{n \geq 1} d_{2 n+1} \frac{z^{2 n+1}}{(2 n+1) !}=\frac{\sin (z)(2-\cos (z))-z}{\cos (z)^{2}} .
$$

We can take the sum of these two equations to obtain $\sum_{n \geq 2} d_{n} \frac{z^{n}}{n !}$, but there seems to be no particular simplification. The first values of $\bar{d}_{n}$ for $n \geq 2$ are as follows:

$$
1,2,7,26,117,594,3407,21682,151853,1160026,9600567 \ldots
$$

And the first values of $d_{n}$ for $n \geq 2$ are:

$$
2,2,12,26,178,594,4792,21682,202374,1160026,12303332, \ldots
$$

4.4. Remaining cases. For the dihedral group, we have:

$$
K\left(I_{2}(m)\right)=\left\{\begin{array}{l}
1 \text { if } m \text { is odd } \\
2 \text { if } m \text { is even. }
\end{array}\right.
$$

Among the exceptional groups, $E_{6}$ is the only one where the longest element is not central. We apply Equation (4) and the calculation is the following:

$$
\begin{aligned}
K\left(E_{6}\right) & =K\left(D_{5}\right)+K\left(A_{4} \times A_{1}\right)+\frac{1}{2} K\left(A_{2} \times A_{1} \times A_{2}\right)+K\left(A_{5}\right) \\
& =26+25+15+16=82 .
\end{aligned}
$$

The first two terms correspond to the terms where $s_{i} \neq s_{j}$ and $w_{0} s_{i} w_{0}=s_{j}$. The third term corresponds to a fixed point of the graph automorphism, the vertex of degree 3. It is treated using the second part of Proposition 3.5. The fourth term corresponds to the other fixed point of the graph automorphism, it is treated using the first part of Proposition 3.5.

For all the remaining groups, the longest element is central and we can apply Equation (3). This gives:

$$
\begin{aligned}
K\left(H_{3}\right) & =K\left(I_{2}(5)\right)+K\left(A_{1} \times A_{1}\right)+K\left(A_{2}\right)=4, \\
K\left(H_{4}\right) & =K\left(H_{3}\right)+K\left(I_{2}(5) \times A_{1}\right)+K\left(A_{2} \times A_{1}\right)+K\left(A_{3}\right)=12, \\
K\left(F_{4}\right) & =K\left(B_{3}\right)+K\left(A_{2} \times A_{1}\right)+K\left(A_{1} \times A_{2}\right)+K\left(B_{3}\right)=16 .
\end{aligned}
$$


Eventually, we have:

$$
\begin{aligned}
K\left(E_{7}\right)= & K\left(E_{6}\right)+K\left(D_{5} \times A_{1}\right)+K\left(A_{4} \times A_{2}\right)+ \\
& K\left(A_{3} \times A_{1} \times A_{2}\right)+K\left(A_{1} \times A_{5}\right)+K\left(D_{6}\right)+K\left(A_{6}\right) \\
= & 82+156+75+120+96+178+61=768,
\end{aligned}
$$

and

$$
\begin{aligned}
K\left(E_{8}\right)= & K\left(E_{7}\right)+K\left(E_{6} \times A_{1}\right)+K\left(D_{5} \times A_{2}\right)+K\left(A_{4} \times A_{3}\right)+ \\
& K\left(A_{2} \times A_{1} \times A_{4}\right)+K\left(A_{6} \times A_{1}\right)+K\left(D_{7}\right)+K\left(A_{7}\right) \\
= & 768+574+546+350+525+427+594+272=4056 .
\end{aligned}
$$

\section{FinAL REMARKS}

Let us briefly mention some related results that will appear in [7]. Let $c$ be a Coxeter element for $W$, and consider the set of noncrossing partitions $\mathcal{P}^{N C}(W, c)$ (see [3] for background on noncrossing partitions). This sets naturally embeds in $\mathcal{P}(W)$. It is not stable under the action of $W$, but we can consider how it is divided in equivalence classes (each class is the intersection of $\mathcal{P}^{N C}(W, c)$ with a $W$-orbit). There are $K(W)$ equivalence classes. We will show in [7] that we can in some sense compute the cardinality of each class, and that this leads to hook length formulas in type A and B.

Let us end this article which a more general question, which is not very precisely stated. Let $G$ be a subgroup of $G L\left(\mathbb{R}^{n}\right)$ (there are probably some restrictions to consider, see below). We can define a set

$$
\mathcal{P}(G)=\left\{\pi \subset \mathbb{R}^{n}: \exists g \in G, \pi=\operatorname{Fix}(g)\right\},
$$

and let $\mathcal{M}(G)$ denote the set of maximal chains in $\mathcal{P}(G)$ with respect to inclusion. The group $G$ acts on $\mathcal{P}(G)$ and $\mathcal{M}(G)$, and we can define let $K(G)=\#(\mathcal{M}(G) / G)$. We have examined the case where $G$ is a finite reflection group but we see that the definition is valid in a more general context. Note that a natural restriction on the group $G$ is the requirement that $\mathcal{M}(G)$ is a set of complete flags. Suppose for example that $G$ is the set of invertible upper-triangular matrices. Then $\mathcal{P}(G)$ is the set of all linear subspaces of $\mathbb{R}^{n}$, as can be seen using the $L U$ decomposition. So $\mathcal{M}(G)$ is the complete flag variety $G L\left(\mathbb{R}^{n}\right) / G$. Using the Bruhat decomposition, we see that $K(G)=n$ !. It might be of interest to examine the case of other groups.

\section{REFERENCES}

[1] D. André: Développement de sec $x$ and tg $x$, C. R. Math. Acad. Sci. Paris 88 (1879), 965-979.

[2] V.I. Arnol'd: The calculus of snakes and the combinatorics of Bernoulli, Euler, and Springer numbers for Coxeter groups. Russian Math. Surveys 47 (1992), 1-51.

[3] D. Armstrong: Generalized Noncrossing Partitions and Combinatorics of Coxeter Groups. Mem. Amer. Math. Soc. 202, 2009.

[4] D. Armstrong, V. Reiner and B. Rhoades: Parking spaces. Preprint (2012), available at arXiv:1204.1760. 
[5] A. Björner and F. Brenti: Combinatorics of Coxeter groups. Graduate Texts in Math., Vol. 231, Springer, New York, 2005.

[6] J.E. Humphreys: Reflection Groups and Coxeter Groups. Cambridge University Press, Cambridge, 1990.

[7] M. Josuat-Vergès, Refined enumeration of noncrossing chains and hook formulas, in preparation.

[8] M. Josuat-Vergès, J.-C. Novelli, and J.-Y. Thibon: Algebraic combinatorics on snakes. J. Combin. Theory Ser. A. 119(8) (2012), 1613-1638.

[9] N. Reading: Chains in the noncrossing partition lattice. SIAM J. Discrete Math. 22(3) (2008), $875-886$.

[10] K. Saito: Principal Г-cone for a tree, Adv. Math. 212(2) (2007), 645-668.

[11] T.A. Springer: Remarks on a combinatorial problem. Nieuw. Arch. Wisk. 19(3) (1971), 30-36.

[12] R.P. Stanley: Some aspects of groups acting on finite posets. J. Combin. Theory Ser. A 32 (1982), 132-161.

[13] R.P. Stanley: A survey of alternating permutations. Contemp. Math. 531 (2010), 165-196.

CNRS and Institut Gaspard Monge, Université Paris-Est Marne-la-Vallée, 5 Boulevard Descartes, Champs-Sur-Marne, 77454 Marne-la-Vallée cedex 2, France

E-mail address: matthieu.josuat-verges@univ-mlv.fr 